\title{
HISTORY? MY STORY! \\ Ein Plädoyer für das ICh in Pop-Geschichte
}

\section{Dietrich Helms}

Die Geschichte der Pop- und Rockmusik beginnt 1972 mit »Crazy Horses« von The Osmonds - wenn ich mich recht erinnere. Wir Kinder liebten es, den Hook des Stücks, ein extrem hohes Kreischen nach einer zuvor eher tiefen Gesangsstimme, zu imitieren. Das Stück war in vielen europäischen Charts in den Top-Ten und wir hörten es mehrfach auf dem Sender, den unsere Eltern im Radio eingestellt hatten. Ich erlebte das erste Mal, dass Musik etwas Wildes, aber auch Groteskes und an den Nerven Sägendes sein konnte, dass es Spaß macht, aus voller Lunge und vollem Herzen mitzuschreien. ${ }^{1}$

Aus demselben Jahr stammt eine weitere, sehr lebhafte frühe Erinnerung, in der ein Banjo spielender Hund auf einem roten Kissen eine zentrale Rolle spielt, der von seinem Wunsch nach einer Miezekatze sang. Seine Single, die wochenlang die bundesdeutschen Single-Charts anführte, wurde zur Nummer Eins meiner Plattensammlung. J ahre später kamen die Platten, von denen ich weiß, dass sie in den Sammlungen vieler Mit-Babyboomer stehen: Mike Oldfields Tubular Bells (1973), Santanas Greatest Hits (1974), Supertramps Crime Of The Century (1974), Watch von Manfred Mann's Earth Band (1977) und einige mehr. Mädchen, die ich besuchte, hatten Simon and Garfunkel's Greatest Hits (1972) auf ihren Plattentellern. Was mir heute auffällt: All diese Platten - mit Ausnahme von Watch - waren zu der Zeit, als sie um 1978 in meinen sich nur langsam füllenden Plattenständer und gleichzeitig in die Sammlungen meiner Freunde kamen, bereits einige J ahre alt. Etwas Ähnliches bildet meine Plattensammlung um 1982 mit Absolutely Live von den Doors (1970) ab. Ich kann mir dieses Phänomen nur so erklären, dass es so etwas wie einen Generationenkanon von Klassikern gab, dessen Zusammenstellung und Fixierung vier bis fünf J ahre, teilweise auch länger dauerte. Auch heute noch haben diese Platten für mich einen gewissen Wert, der unabhängig von der Zeit ist - im Gegensatz zu den meis-

1 Was meine Erinnerung als wiehernden Schrei des Sängers überliefert, wurde mit einer Yamaha YC-30 Comboorgel mit Portamentostrip produziert. 
ten LPs, die ich kaufte, weil ich ein Stück aus den aktuellen Charts darauf gut fand. Barcley J ames Harvests Gone To Earth von 1977 z. B. hatte ich auf Kassette. Das Geld reichte nur für die wirklich wichtigen Platten. In den frühen 1980er J ahren wuchs die Sammlung schneller - zahlenmäßig und stilistisch: für eine kurze Zeit Ougenweides Mittelalterfolkrock und deutsche Liedermacher (die Friedensbewegung!), dann Avantgardejazz, -funk, -rock: Material, Slickaphonics, James Blood Ulmer (das Moerser J azz Festival!), etwas verspätet dann am Rande der Punk und nach dem Abitur schließlich die Klassik (das Studium!).

Wenn ich jetzt durch die verschiedenen Geschichten der populären Musik blättere, kommt die Musik meiner frühen Geschichte darin nicht vor. Mit Wum alias Loriot und The Osmonds hätte ich auch nicht gerechnet, aber »Tubular Bells« und »Black Magic Woman«, »Dreamer« oder »Davy's On The Road Again«? Meine Geschichte beginnt nicht wie diese Bücher mit den ersten Blues-Aufnahmen um 1920; Mamie Smith und Robert J ohnson spielten für mich nie eine Rolle. Erst Anfang der 1980er J ahre macht meine Plattensammlung einen kurzen Abstecher zu J ohn Lee Hooker und The Best Of The Blues. Der vermeintliche Schöpfungsakt des Rock 'n' Roll im J ahre 1955 hat für mich nie eine Rolle gespielt. Diese Geschichte meiner Eltern verstehe ich nicht als Teil meiner eigenen.

Was hat das alles mit einer Geschichte der Pop- und Rockmusik zu tun? Grundsätzlich ist von keiner Historiographie zu verlangen, dass sich jeder Einzelne in ihr wiederfindet. Es erhöht allerdings die Akzeptanz einer Geschichte der jüngeren Vergangenheit, wenn sie zumindest zum Teil auch das Leben derjenigen berührt, die dabei gewesen sind. Die allgemeine Musikgeschichte musste diese Erfahrung gerade wieder machen bei der Aufarbeitung der Geschichte der DDR und der Diskussion darüber, wer eigentlich eine solche Geschichte schreiben darf. Ich möchte hier behaupten, dass die Geschichtsschreibung der populären Musik mehr deutlich gemachte Subjektivität braucht, um als intersubjektiv gültig und vielleicht sogar als objektiv wahr anerkannt zu werden.

Die Popularmusikforschung hatte in ihrer Vergangenheit einiges mit ihrem Gegenstand gemein. Auch sie verstand sich einmal als Gegenentwurf - in ihrem Fall zur traditionellen Musikwissenschaft. Sie begehrte auf gegen kulturelle Hegemonien und fremdbestimmte Bildungskanons, wollte jugendlichen Subkulturen zu ihrem Recht und zur Würde eines wissenschaftswerten Gegenstands verhelfen. Inzwischen ist sie - ebenfalls wie die populäre 
Musik - »in der Mittel der Gesellschaft « angekommen und ein etablierter Teil der Kultur und ihrer Erforschung geworden. Was mich angesichts der widerständigen Anfänge erstaunt: Die Popularmusikforschung geriert sich, gerade was das Schreiben von Geschichte betrifft, inzwischen nicht selten genauso wie das, was sie am Anfang bekämpfte: US-amerikanische Autoren schreiben Nationalgeschichten der populären Musik, ohne einen Blick auf Einflüsse und Ursprünge außerhalb der eigenen Grenzen zu werfen (s. Martin Pfleiderer in diesem Buch, S. 61, und erinnern mich stark an die deutsche Musikgeschichtsschreibung des 19. Jahrhunderts, die eifrig die Heldengeschichte einer Kultur betrieb, die sie als nationales, deutsches Merkmal prägen wollte. Gleichzeitig werden in einschlägigen Zeitschriften Kanons formuliert, die den Musikgeschmack »höher gebildeter weißer Männer des zweiten Lebensdrittels« (v. Appen/Doehring/Rösing 2008: 37) als »die besten Songs aller Zeiten« (www.poplist.de) absolut setzen. Und selbstverständlich werden orthodox die alten Schöpfungsmythen gepflegt (»afroamerikanische Wurzeln«, »1955«, »1968«, »Widerstand«, »Subkultur«...), statt erst einmal allen Selbstverständlichkeiten zu misstrauen, was konsequent wäre, wenn man dem Mythos des Rock und seine angebliche Widerständigkeit ernst nähme. Auch wenn die Etymologie von »Rockmusik« nicht endgültig zu klären ist, von Fels und Versteinerung ist in keinem Erklärungsversuch etwas zu lesen, vielmehr sind Steine im Rock immer rollende.

Übernehmen wieder einmal die Alten, Arrivierten und Staatstragenden die Definitionsmacht und formen die Geschichte der populären Musik nach dem Vorbild dessen, was sie einst als Hochkultur abgetan haben? Die ersten Hörer der Rolling Stones überschreiten zur Zeit gemeinsam mit ihrer Band die 70. Da ist es verständlich, dass man zurück blickt, nach Sinn in der eigenen Geschichte sucht und sich freut, anders gewesen zu sein als »die J ugend von heute «, dass man die eigene Musik auf den Sockel hebt und ihr historischen Wert verleihen möchte. Es tut gut zu lesen, dass die eigene Hörbiographie der offiziellen Geschichte entspricht und dass die Plattensammlung ziemlich genau den Kanon wiedergibt. Doch es ist weniger das Lebensalter der Geschichtsfestschreiber, das ich mit den »Alten und Arrivierten« meine, sondern die Art und Weise, mit der so oft Geschichte geschrieben wird - nach Altväterart: Ohne erkennbare Zweifel am eigenen Tun, ohne dem Leser zu verdeutlichen, was das denn eigentlich für ein Ding ist, dessen Geschichte man schreibt, ohne die Filterprozesse offen zu legen, die Geschichtsschreibung bedeutet, und dann so oft in Form einer Universalgeschichte, die alles in mundgerechten Happen in einen großen Topf wirft und umrührt, sodass alles mit allem zusammenzuhängen scheint und doch nur nebeneinander wie Gemüse und Fleisch in der Suppenterrine schwimmt, 
mit Wasser als verbindendem Element. Mit demselben hegemonialen Gebaren, das man einst mit der so genannten Hochkultur bekämpfte, werden Mythen verfestigt und Kanons durchgesetzt, in Lehrbücher gepresst und in Schulen und Universitäten als zu lernendes Wissen verbreitet - nur geht es nicht mehr um Bach, Beethoven, Wagner, sondern um Beatles, Bob Dylan und Zappa.

Bräuchte es nicht neben dem Schreiben von Geschichten eine ebenso seitenstarke Diskussion darüber, was das eigentlich sein könnte, eine »Geschichte der populären Musik«, und ob beziehungsweise worin sie sich unterscheiden sollte von den Geschichten der Musik, die keine populäre Musik ist? So werden zum Teil auch Lehren aus Fehlern ignoriert, die die Geschichtsschreibung der Kunstmusik längst gezogen hat und das, obwohl auch sie die Diskussionen in der Geschichtswissenschaft eher verspätet rezipiert und ihr eigenes Tun erst in den letzten J ahren verstärkt reflektiert (vgl. z. B. Danielczyk u.a. 2012). Wenn wir populäre Musik noch als etwas anderes ansehen als Kunstmusik, sollten wir auf jeden Fall nicht aufhören, diese Unterschiede zu diskutieren, denn es könnte sein, dass das Ergebnis einer solchen Diskussion die Überzeugung ist, dass die Andersheit der populären Musik auch eine andere Methodik der Beschreibung ihrer Geschichte braucht.

Nach den Diskussionen um eine Definition der populären Musik in den 1980er und 1990er J ahren wurde das Problem weitgehend ad acta gelegt. Das Phänomen ist inzwischen gealtert, es haben sich implizite Kanons gebildet, es ist ein paradigmatisches Wissen vom Gegenstand im Sinne Thomas Kuhns (1976) entstanden, auf dessen Basis sich durchaus sinnvoll wissenschaftlich arbeiten lässt. Doch ist es gefährlich, Geschichte zu schreiben, ohne zu reflektieren, woher die Setzungen stammen, aus denen ihr Gerüst hergestellt werden soll. Es gibt in der Historiographie noch keine Riesen, auf deren Schultern der gegenwärtige Forscher beruhigt um die Stabilität des von den Vorgängern gesammelten wissenschaftlichen Wissens stehen könnte.

Geschichte ist ein Filterprozess. In der älteren Geschichte haben frühere Generationen die Aufgabe der Auswahl übernommen: Sie haben Kompositionen nicht zum Druck angenommen, Manuskripte verbrannt, Bibliotheken zerstört oder einfach Lieder nicht mehr gesungen, Noten nicht mehr abgeschrieben. In der neuesten Geschichte dagegen ist noch so viel da, nicht nur weil die Zeit noch kaum Gelegenheit hatte, die Archive zu zerstören, sondern auch weil in den vergangenen J ahrzehnten so viel gespeichert wurde wie nie zuvor und weil die neuen Medien trotz schneller Medienwechsel nicht mehr vergessen. Filterprozesse werden daher umso stärker von Einzel- 
nen verantwortet, denn es gibt keine Tradition der Filter - zumindest nicht in der Wissenschaft. Auch deshalb sollte der Historiker transparent machen, warum er sich für ein konkretes Beispiel, eine bestimmte Stilrichtung oder ein bestimmtes soziales Phänomen entscheidet, wenn er Pfade durch das chaotische Dickicht des gerade Vergangenen schlägt. Gerade wenn man eine Vergangenheit rekonstruiert, die erst seit kurzer Zeit Geschichte ist, sollte man erwarten, dass der Autor seine Leser mit einer argumentativ gut begründeten Auswahl überzeugt und nicht mit seiner Autorität als Autor eines Buchs, als Professor, Musikkritiker oder selbsternannter Szenekenner. Ich erwarte, dass er »ich « sagt, wenn er unter den tausend ähnlichen Stücken ein Beispiel auswählt, weil er es besonders gut kennt oder gerne mag, und dass er mir erklärt, warum er eine bestimmte Band in die Geschichte aufnimmt und eine andere nicht. Ich erwarte, dass er statt eines positivistischen »das war so « die Subjektivität, die hinter einer Auswahlentscheidung steht, erkennen lässt und mir damit die Chance lässt, eben diese Subjektivität seiner Erzählung zu erkennen, dass er deutlich macht, dass es auch andere Wege der Auswahl, andere Perspektiven auf die vergangene Zeit gibt, die dasselbe Recht auf Geschichtsmächtigkeit haben. Wenn Pop und Rock einmal gegen das Establishment revoltiert haben, gegen kulturelle Hegemonien, sollten ihre Historiker - die diese Mythen ja gern weiter pflegen - hier besonders sensibel sein.

Zum Bekenntnis der Subjektivität gehört auch, dass man die eigene Definition des Gegenstands seiner Geschichte offen legt, denn sie beeinflusst vorrangig alle Filterprozesse. Ich habe bewusst formuliert, dass man die »eigene Definition « offen zu legen habe, und nicht, dass man den Gegenstand definieren müsse, denn in der Tat: »It is difficult to come up with a satisfactory definition of >popular music« (Starr/Waterman 2010: 2) und »der Versuch, Pop zu definieren, wird letztendlich in allen Anläufen scheitern« (Seifert 2007: 15). Doch mit solchen Aussagen im Vorwort einer Popoder Rockgeschichte wirft man lediglich Nebelkerzen, die verschleiern, dass hinter allem eben doch eine ganz eindeutige Idee von der Geschichte der Rock- und Popmusik versteckt ist. Woher sollte sonst diese Selbstgewissheit stammen, die eine Geschichte der einen Rockmusik erzählen zu können? Wäre der Gegenstand nicht einzugrenzen, könnte man eine Geschichte überhaupt nicht schreiben. Man entzieht sich mit solchen pauschalen Aussagen allerdings geschickt der Kritik und entschuldigt gleichzeitig die Subjektivität der Auswahl, ohne sie begründen zu müssen und ohne sie als subj ektiv transparent machen zu müssen.

Eine eindeutige Aussage darüber, was der Autor selbst unter »populärer Musik« versteht, ist jedoch ein doppeltes Muss für eine Geschichte, um dem 
Leser Filterprozesse deutlich zu machen. Versteht man populäre Musik als kulturellen Ausdruck von »denen da unten«, entsteht eine ganz andere Geschichte mit ganz anderen Anfängen und einem anderen Verlauf als eine populäre Musik, die als industriell gefertigt und massenmedial verbreitet verstanden wird, und auch die Stücke und Bands, die als Stationen einer solchen Geschichte zitiert werden, sind andere. Es macht einen Unterschied, ob man eine Geschichte vom Widerstand der Subkulturen schreibt, deren Verzweigungen immer dann enden, wenn sich der Mainstream eine Strömung einverleibt, oder ob man eine Geschichte entlang der größten Hits auf den obersten Plätzen der Charts schreibt. Selbstverständlich kann man auch eine Geschichte schreiben, die auf der Dialektik von Subkultur und Mainstream oder von Individuation und Proliferation (Helms 2008) beruht. Das ergäbe allerdings wieder andere Filterprozesse mit anderen Bands und anderen Stücken, die Geschichte machen (vgl. hierzu ausführlicher Helms 2011).

Viele Geschichten des Pop oder Rock lesen sich dagegen wie ein Sammelsurium: Ein wenig Sozialgeschichte der Jugend oder der Unterschicht, ein wenig Geschichte afro-amerikanischer Musik, ein wenig Biographik, ein wenig Technologiegeschichte, ein wenig Wirtschaftsgeschichte, ein wenig Gender und manchmal geht es auch um Musik. Doch da es bis vor Kurzem nur wenige Auseinandersetzungen mit Methoden einer musikanalytischen Beschreibung populärer Musik gegeben hat und deren Tauglichkeit für die Konstruktion einer Stilgeschichte z.B. noch kaum erprobt sind, fallen diese Abschnitte meist eher kurz aus. Zusammengehalten - wenn überhaupt wird das Ganze durch einen impliziten Kanon von Stücken, den die Autoren zugrunde legen, über dessen Existenz sie jedoch in der Regel keine Rechenschaft ablegen. Auch hier gilt das Prinzip des Zufalls: Mal sind es Stücke, die als Beispiele für Widerständigkeit oder den Ausdruck der unterdrückten Unterschicht dienen, mal solche, die künstlerisch wertvoll genannt werden können, mal andere, die die größten Verkaufsschlager ihrer Zeit waren.

Woher kommt das? Machen wir einmal das gedankliche Experiment, Geschichten einzelner Aspekte des »Populären« zu schreiben: Sozialgeschichten, Technologiegeschichten, Gendergeschichten, Geschichten von Rassekonstruktionen, Wirtschaftsgeschichten, Verkaufserfolgsgeschichten und Originalitätserfolgsgeschichten... Da in diesen Geschichten Gesellschaft in ihrer Funktionalisierung von Musik, die Mediatisierung der Musik durch Technik, die Nutzung von Musik in Prozessen der Geschlechter- oder Rassenzuschreibungen, die Vermarktung von Musik oder die Frage kunstvoller Komplexität der musikalischen Struktur im Mittelpunkt stehen, wird man bald erkennen müssen, dass es schwer fällt, eine Begrenzung auf die populäre 
Musik zu rechtfertigen. Weder eine Geschichte musikalischer Technologie noch eine historische Auseinandersetzung mit Geschlechterverhältnissen in der Musik, weder eine Geschichte der Musikwirtschaft noch eine von widerständiger Musik rechtfertigt eine Trennung in Kunstmusik und populäre Musik. Wenn man Kunstmusik und populäre Musik nicht als mehr oder weniger getrennte Mengen von Stücken versteht, sondern als Diskurse um komponierte und um performte Musik, kann man beim Schreiben einer Geschichte musikalischer Performance ein Phänomen wie Madonna nicht von einem anderen wie Anna Netrebko abtrennen. Dasselbe gilt selbst für analytische Annäherungen an populäre Musik, wie z.B. Richard Middleton an einem Beispiel deutlich macht: »One could not satisfactorily analyse modalism in rock music without also dealing with the decline of modal folk song, in its traditional social contexts; the urban folk revival; the use of modal techniques by elite composers, and the >discovery of modal medieval and renaissance music (Middleton 1990: 12).

Wenn allerdings die einzelnen Kategorien, aus denen sich das Paradigma der populären Musik zusammensetzt, nichts zu der Spezifik einer Geschichte der populären Musik beitragen, was ist dann das Spezifische einer Geschichte der Pop- und Rockmusik? Reebee Garofalos Rockin' Out. Popular Music in the USA (2008) z. B. behandelt Technologien, Massenmedien, Konstruktionen von Geschlecht und Rasse, Crossover-Phänomene, Widerstand und Kommerzialisierung, politisches Engagement, Feminismus, einzelne Genres, Stile, Musiker, Songs, Analysen... Man probiert die Vergangenheit in bunten Häppchen und ist beeindruckt von der Fülle dessen, was hier ausgebreitet wird. Anhand der Breite der Argumentation und der vielen Daten erscheint populäre Musik als komplexes Gebilde und es wird verdrängt, dass jeder einzelne Punkt nicht allein typisch für populäre Musik ist, dass die Basis dieser Geschichte keine gemeinsame Eigenschaft ihrer Gegenstände ist, außer einer: Sie alle gehören einer Menge von Songs und ihrer Produzenten an, von der der Autor behauptet, sie mache z.B. die "popular music in the USA Wenn populäre Musik ein multikategoriales Feld ist, wie Middleton (1990) es beschreibt, können es nicht die Kategorien sein, die beschreiben, welche Songs dazu gehören und welche nicht (denn von Song zu Song sind andere Kategorien gültig), sondern umgekehrt: die Gewissheit, welche Stücke auf jeden Fall dazugehören, bringt die Wissenschaft in die Verlegenheit, eine Vielzahl von Kategorien zur Beschreibung des Feldes heranzuziehen.

Für den Leser ist die Eingrenzung der Gegenstands auf der Basis eines Kanons sicherlich die am schwierigsten zu überprüfende, da sie sich analytisch nicht aus dem einzelnen Gegenstand ergibt. Wessen Kanon hier zugrunde gelegt wird und wie er entstand, wird nicht transparent gemacht. 
Man muss dem Autor vertrauen, dass er weiß, was er tut. Hierdurch entsteht ein deutliches Machtgefälle zwischen Autoren und Lesern. Akzeptiert man die Auswahl, akzeptiert man auch die Eigenschaften des Gegenstands, die an ihr exemplifiziert werden. Die dargestellten Sachverhalte werden damit zu objektiven Daten, zu einer positivistischen historischen Wahrheit erhoben. Ich möchte noch einmal betonen, dass es ganz und gar nicht verwerflich, sondern sogar notwendig ist, das große Total des kollektiven Gedächtnisses zu filtern, doch sollte der Leser die Chance haben, die Prozesse zu hinterfragen, die zu einer Auswahl führen. Gerade in einer so jungen Disziplin wie der Geschichtsschreibung der populären Musik sollte er beim Machen der Geschichte zuschauen dürfen, wenn man aufgeklärt Wissenschaft treiben will.

Mir macht die dargestellte Art der Geschichtsschreibung immer wieder deutlich, dass populäre Musik an sich wissenschaftlich - je nach theoretischem Hintergrund - nur als Diskurs bzw. als soziales System zu beschreiben und zu fassen ist. Das kann in letzter Konsequenz allerdings nur bedeuten, dass eine wissenschaftliche Geschichte der populären Musik nur eine Diskurs- oder System- bzw. eine Begriffsgeschichte sein kann, eine Geschichte, die davon handelt, wie durch Kommunikation das Populäre vom Nicht-Populären abgegrenzt wird, wie soziale Gruppen darum streiten, Begriffe wie populäre Musik, Jazz, Pop oder Rock und ihre Inhalte definieren zu dürfen, und wie sie mit diesen Grenzen umgehen.

Eine in diesem Kontext zu bedenkende Arbeitsgrundlage einer Popularmusikforschung ist die Tatsache, dass ihre Paradigmen und Kanons - anders als z. B. in der Mathematik oder der theoretischen Physik - zum großen Teil außerhalb des Systems der Wissenschaft entstehen. Der Wissenschaftler in seiner Rolle als Wissenschaftler ist an der Kanonbildung nicht beteiligt, er kann - zugespitzt formuliert - überhaupt nicht wissen, was aktuell populäre Musik ist. In so fern hat er keine Macht über die eigenen Begriffe. Er kann einem Punk nicht ernsthaft sagen wollen, was Punk ist und welches die wichtigen Stücke der Geschichte des Punk sind. Er kann als Euroamerikaner auch einem Afroamerikaner kaum mit hinreichender Autorität die eigene Musikgeschichte erklären oder als mittelalter Hochschuldozent einem J ugendlichen etwas über die Musik der Jugend - wenn er denn (diese Kategorie hatten wir bisher noch nicht erwähnt) Popgeschichte als Geschichte der Nutzung von Musik durch Jugendliche schreiben will. Wenn allerdings populäre Musik ein Diskurs ist und wenn nicht die Wissenschaft, sondern die Rezipienten festlegen, was wichtig ist, was ein Klassiker (weil stilbildend oder besonders originell) oder Evergreen (weil über viele J ahre hin kommerziell erfolgreich) und welche Bands wichtig sind, warum schreibt Wissen- 
schaft dann keine Rezeptionsgeschichte, keine Geschichte aus der Perspektive der Hörer? Warum tut sie es der traditionellen, zunehmend überholten Geschichtsschreibung der Kunstmusik nach, die ebenfalls von einem Werkekanon ausgeht, allerdings - durch Anwendung anerkannter Analyseverfahren - ihren Kanon viel einfacher, viel einheitlicher und noch dazu in größerem Maße selbstbestimmt begründen kann. Sollte man nicht für die »andere« Musik, die populäre Musik sein will (solange sie noch Grenzen zur Kunstmusik aufbaut), statt von apriori als wichtig angenommenen »Werken« auszugehen, eine Geschichte schreiben, die schildert, wann und wie welche Stücke warum für wen wichtig wurden?

Wenn Kanons und Paradigmen außerhalb der Wissenschaft entstehen, wird der Wissenschaftler zum Beobachter und muss empirisch arbeiten. Er darf Kanons und großen Mythen nicht unhinterfragt zur Grundlage seiner Geschichte machen, sondern muss sie durch Quellenstudium belegen und damit zunächst in die Wissenschaft hinein holen. Was passiert, wenn man tatsächlich einmal an die Quellen geht, belegen u.a. die Arbeiten von Eliah Wald (z.B. in diesem Band). Da viele Zeitzeugen heute noch leben, muss der Historiker hinausgehen und fragen. Die Tatsache, selbst einmal jung und dabei gewesen zu sein (s. z. B. das Vorwort von Garofalo 2008: ix), reicht mir nicht als Autorisierung für die Abfassung einer wissenschaftlichen Universalgeschichte des Rock. Die ehrlichsten Rock- und Popgeschichten auf dem Markt sind nach meinem Empfinden meist biographisch angelegt; sie beschreiben offen ein individuelles Leben mit der Musik und keine Geschichten, die das Ich hinter einem pseudo-wissenschaftlichen unpersönlichen Stil verbergen. Hier kann ich mit voller Überzeugung sagen: »] a so war das damals bei uns«, oder »Das habe ich damals anders empfunden«. Warum fragt man nicht die vielen Ichs, aus denen das kollektive Gedächtnis besteht und wertet die Antworten nach bestem Wissen mit empirischen Methoden aus? Warum fragt die Wissenschaft nicht diejenigen, die die Kanons - und damit auch die Geschichte - machen?

Ein Beobachter (auch ein wissenschaftlicher), der Erinnerung (re-)konstruiert, unterscheidet mindestens zwei Perspektiven: eine kollektive und eine individuelle. J e näher der Zeitraum eines Ereignisses, das erinnert werden soll, an der Gegenwart liegt, desto wirkmächtiger ist die individuelle Perspektive oder eine kollektive Perspektive des Nahbereichs, die ein Individuum und seinen unmittelbaren sozialen Kontext umfasst. Populäre Musik, so möchte ich behaupten, war immer wichtiger für die Konstruktion von individueller Geschichte bzw. der kollektiven Geschichte eines sozialen Nahbereichs als für eine größere kollektive Geschichte. Sie ist maximal Generationengeschichte und zumindest in Europa kein Teil der Nationalgeschich- 
te. Wenn die Ergebnisse der empirischen Forschung stimmen, wird populäre Musik von ihren Rezipienten auf sozialer Ebene vorwiegend zur Individuation und Gruppenbildung funktionalisiert (s. z. B. Rösing 1992). Damit sind individuelle Hörgeschichten (bzw. Hörgeschichten kleinerer sozialer Gruppen) für den Einzelnen relevanter als Musikgeschichten mit universalem Anspruch.

Die Bedeutung der Individualgeschichte verstärkt sich auch durch die mediale Überlieferungssituation. Im Internet ist so gut wie jeder Song zu jeder Zeit verfügbar. Keine Schallplattenauflage ist mehr abverkauft, keine CD nicht lieferbar. Dazu kommt kein Song mehr zusammen mit Markern seines Alters: eine MP3-Datei hat kein lesbares Copyrightdatum und kann nicht mehr zerkratzen. Wann etwas entstanden ist, wird zunehmend weniger wichtig gegenüber dem Datum, an dem es von Einzelnen oder einer bestimmten Gruppe im end- und zeitlosen Datenmeer der Neuen Medien entdeckt wurde. So gehört Paul Potts »Nessun dorma « zur Musikgeschichte des J ahres 2007 und längst nicht mehr ins J ahr der Uraufführung von Puccinis Turandot 1926. Und Udo Jürgens »Griechischer Wein « klingt für viele längst nicht mehr nach 1974, als das Lied veröffentlicht wurde, sondern nach dem Schlagerrevival der späten 1990er und für einige Schülerjahrgänge der 2000er nach ihrer Abiturfeier. Auch weil Titel jeder Zeit wieder aktualisiert und zu Symbolen für eine andere, neuere Zeit gemacht werden können - von kleinen Gruppen oder von ganzen Generationen - ist eine an Produktionsdaten orientierte Geschichte problematisch.

Individuelle Hörgeschichten müssen nicht der Chronologie der Produktion entsprechen; ihr roter Faden besteht nicht aus Verwandtschaften der Stilistik, Lehrer-Schülerverhältnissen von Musikern, Reihenfolgen von Produktion oder Charts-Notierungen, dem Fortschritt des musikalischen Materials, sondern aus dem Lebenslauf eines Hörers, an dessen erinnerungswerten Ereignissen sich einzelne Stücke anheften und schließlich symbolisch für diese stehen: »die Party zum 16. Geburtstag«, »unser Abi-Lied«, »das habe ich gerade gehört, als die Nachricht vom Anschlag auf das World Trade Center kam «... Populäre Musik ist genau deshalb so bedeutungsoffen konstruiert (Helms 2004), damit sie diese Funktion der individuellen Aneignung erfüllen kann. Die wirkliche Geschichte der populären Musik, so meine ich, entsteht genau aus diesen individuellen Hörgeschichten. Ein einzelner Hörer, ein Liebes- oder Freundespaar, eine Clique, die Jugendlichen einer Stadt oder gar eine ganze Generation erlebt etwas mit Musik, was diese Musik zu ihrer eigenen macht. Songs werden so zu Symbolen einer Zeit und übrigens nicht, weil sie z.B. im Text auf Ereignisse der Zeitgeschichte verweisen. Für mich, und ich denke auch für etliche meiner Freunde der Abiturzeit, stehen die Doors nicht für Vietnam und die späten 1960er, son- 
dern für die fatalistische Stimmung der Zeit der Umsetzung des NATO-Doppelbeschlusses 1982/83 und die Angst vor einem Atomkrieg: »This is the end, my only friend, the end... «. Zu dieser Zeit kam Absolutely Live in meine Plattensammlung.

Wenn Geschichte eine wissenschaftliche Methode ist, durch das Erzählen von Geschichten entlang historischer Daten ${ }^{2}$ die Gegenwart verständlicher zu machen, helfen mir die Popgeschichten auf dem Büchermarkt kaum dabei, mein Hier und J etzt zu ordnen. Ich brauche keine Nationalgeschichte der populären Musik. Ich brauche auch keine Heroengeschichte der USamerikanischen Musik, denn ich habe meine eigenen Helden. Was ich dagegen gerne läse, wäre eine Musikgeschichte einer Generation, z.B. meiner: Erinnert Ihr Euch auch, liebe Babyboomer, an den Hund auf dem roten Kissen und sein Banjo? Welche Platten habt Ihr wann gekauft, was hattet Ihr auf Kassette? Welche Musik assoziiert $\mathrm{Ihr}$ - wenn überhaupt - mit den Demos gegen Pershing und Cruise Missile: »Ein bisschen Frieden«, »Sieben Tage lang«, »Cruise Missiles« oder »The End «? Welche Platten musste man in Eurer Clique in den frühen 1980ern besitzen, um dazu zu gehören? Welche Songs erzählen Eure Lebensgeschichte?

Die Geschichten, die mir vorschweben, sind alles andere als rein subjektive, unwissenschaftliche Erzählungen. Sie arbeiten allerdings mit einer von der historischen Musikwissenschaft selten genutzten Quelle, der oralen Überlieferung, die zunächst einmal zu sammeln und mit empirischen Methoden auszuwerten wäre. Solche Geschichten wären dem Gegenstand angemessen: Sie verstehen populäre Musik als Diskurs, der Geschichte macht und sich durch die Geschichte verändert. Sie gewinnen ihre Gültigkeit offen und erkennbar aus Erinnerungen einzelner Subjekte und nicht aus scheinbar objektiven, weil entpersonalisierten Daten, die doch in ihrer Auswahl zutiefst subjektiv sind. Sie setzen nicht einseitig und autoritär Heroen und einen Kanon fest, schreiben keine Geschichte aus der Perspektive und in der Reihenfolge der Produktion, sondern eben der Rezeption. Sie nehmen diejenigen ernst, die die Geschichte wirklich machen, lauscht sie ihnen ab und schreibt sie ihnen nicht vor. Sie würden die Gegenwart und den vergangenen Weg dorthin erklären helfen und wären nicht nur Lernstoff für Klassenarbeiten und Modulabschlussprüfungen, der durch die Macht eines Älteren bzw. in der Hierarchie höher Stehenden vorgegeben wird. Sie ergäben freilich eine neue, eine populäre Musikgeschichte.

2 »Daten« meine ich im Sinne von lateinisch »datum«, Gegebenes, und damit keine Fakten mit Anspruch auf Realität, sondern Phänomene ( $\mathrm{J}$ ahreszahlen, Ereignisse, Gegenstände, Handlungen...), die wir bei der Konstruktion von Geschichte als intersubjektiv gleichbleibend, anerkannt und gültig voraussetzen. 


\section{Literatur}

Appen, Ralf von / Doehring, André / Rösing, Helmut (2008). »Pop zwischen Historismus und Geschichtslosigkeit. Kanonbildung in der populären Musik. « In: No Time for Losers. Charts, Listen und andere Kanonisierungen in der populären Musik. Hg. v. Dietrich Helms und Thomas Phleps. Bielefeld: transcript, S. 25-49.

Danielczyk, Sandra u.a. (Hg.) (2012). Konstruktivität von Musikgeschichtsschreibung. Zur Formation musikbezogenen Wissens. Hildesheim, Zürich, New York: Olms.

Garofalo, Reebee (2008). Rockin' Out. Popular Music in the USA. Upper Saddle River, NJ : Pearson Education.

Helms, Dietrich (2004). »»l Can't See New York<? Der 11. September und die Bedeutung von >Bedeutung populärer Musik««. In: 9/11 - The world's all out of tune. Populäre Musik nach dem 11. September 2001. Hg. v. Dietrich Helms und Thomas Phleps. Bielefeld: transcript, S. 131-154.

Helms, Dietrich (2008). »What's The Difference? Populäre Musik im System des Pop. « In: PopMusicology. Hg. v. Christian Bielefeldt, Udo Dahmen und Rolf Grossmann. Bielefeld: transcript, S. 75-93.

Helms, Dietrich (2011). »Musikgeschichte für >lange Ohren<? Gedanken zur Geschichtsschreibung nicht nur der populären Musik«. In: Musik und Popularität. Aspekte zu einer Kulturgeschichte zwischen 1500 und heute. Hg. v. Sabine Meine und Nina Noeske. Münster u. a.: Waxmann, S. 25-38.

Kuhn, Thomas (1976). Die Struktur wissenschaftlicher Revolutionen. Frankfurt/M.: Suhrkamp.

Middleton, Richard (1990). Studying Popular Music. Milton Keynes, Philadelphia, PA: Open University Press.

Rösing, Helmut (1992). »Musik als Lebenshilfe? Funktionen und Alltagskontexte«. In: Gesellschaft und Musik. Wege zur Musiksoziologie. Festgabe für Robert $\mathrm{H}$. Reichardt zum 65. Geburtstag. Hg. v. Wolfgang Lipp. Berlin: Duncker \& Humblot, S. 311-331.

Seifert, J ürgen (2007). More than 50 years. Die Geschichte der Rock- und Popmusik. Norderstedt: Books on Demand.

Starr, Larry / Waterman, Christopher (2010). American Popular Music. From Minstrelsy to MP3. New York, Oxford: Oxford University Press.

\section{Abstract}

Many histories of popular music are written with methods similar to and in a comparable vein as histories of art music. They work on the basis of a secret canon of »masterworks«, whose existence and origin is neither made transparent for the readers nor discussed by the authors. This paper argues that, if popular music still makes a difference to art music, its history should be written according to different guidelines. It should be a history of the recipients of popular music, their memories and their lives with music. 